\title{
A Security Assistance System combining Person Tracking with Chemical Attributes and Video Event Analysis
}

\author{
C. Becher ${ }^{a}$, G.L. Foresti ${ }^{b}$, P. Kaul ${ }^{a}$, W. Koch ${ }^{c}$, F.P. Lorenz ${ }^{c}$, D. Lubczyk ${ }^{d}$, C. Micheloni ${ }^{b}$, \\ C. Piciarelli ${ }^{b}$, K. Safenreiter ${ }^{c}$, C. Siering ${ }^{d}$, M. Varela ${ }^{c}$, S.R. Waldvogel ${ }^{d}$ and M. Wieneke $^{c}$ \\ ${ }^{a}$ Department of Natural Sciences, University of Applied Sciences Bonn-Rhein-Sieg, St. Augustin, Germany \\ ${ }^{b}$ Department of Computer Science, Università degli Studi di Udine, Udine, Italy \\ ${ }^{c}$ Department of Sensor Data and Information Fusion, FGAN-FKIE, Wachtberg, Germany \\ ${ }^{d}$ Kekulé-Institute for Organic Chemistry and Biochemistry, University of Bonn, Bonn, Germany
}

\begin{abstract}
Timely recognition of threats can be significantly supported by security assistance systems that work continuously in time and call security personnel in case of anomalous events in the surveillance area. We are describing the concept and the realization of an indoor security assistance system for realtime decision support. The system consists of a computer vision module and a person classification module. The computer vision module provides a video event analysis of the entrance region in front of the demonstrator. After entering the control corridor, the persons are tracked, classified and potential threats are localized inside the demonstrator. Data for person classification are provided by chemical sensors detecting hazardous materials. Due to their limited spatio-temporal resolution, a single chemical sensor cannot localize this material and associate it with a person. We compensate this deficiency by fusing the output of multiple, distributed chemical sensors with kinematical data from laser-range scanners. Considering both the computer vision information and the results of the person classification affords the localization of threats and a timely reaction of security personnel.
\end{abstract}

Keywords: Event Recognition, Behavior Analysis, Person Tracking, Classification, Probabilistic Multiple Hypothesis Tracking (PMHT), Attributes, Security Assistance, Quartz Micro Balance (QMB), TATP, Explosives

\section{INTRODUCTION}

Freedom of movement for people as well as freedom to come together safely in open public events or utilities is vital to each citizen. The defence of this freedom against ubiquitous threats requires the development of intelligent security assistance systems that comprise state-of-the-art surveillance technology and work continuously in time. In our work we demonstrate core functions of an indoor security assistance system for real-time decision support that is based on a heterogeneous sensor suite and multiple sensor fusion techniques. Within this system potential threats are classified, tracked

Further author information: (Send correspondence to M. Wieneke)

$a_{\{\text {christopher.becher, peter.kaul\}@fh-bonn-rhein-sieg.de }}$

$b_{\{\text {michelon, piccia, foresti\}@dimi.uniud.it }}$

$c\{$ w.koch, lorenz, safenreiter, varela, wieneke $\} @$ fgan.de

$d_{\{\text {lubczyk, carsten.siering, waldvogel }\} @ \text { uni-bonn.de }}$ and localized in order to focus the attention of the security personnel.

In many security-relevant utilities, there exist well-defined access regions, e.g. stairways, escalators or gangways. The most efficient way to solve the surveillance task is to focus on these access regions and to continuously monitor and analyze the dynamic events that occur when persons enter the utility. We propose to divide the system into two parts: a videocontrolled area in front of the access region and a scannercontrolled area covering the actual access region (fig. 1).

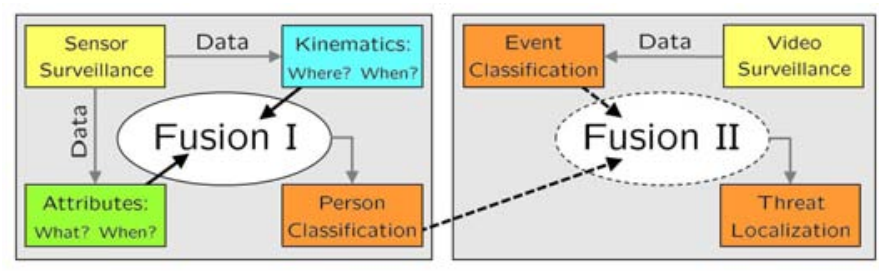

Figure 1. Concept of our security assistance system

The video surveillance module provides event recognition and classification of the events into several alarm levels (fig. 1 - top right). In the explicit event recognition approach, the system has an explicit knowledge of the events that must be identified, and once an event is detected, it can be properly labeled with a semantic description. The fundamental part of an explicit recognition system is thus an a priori knowledge base, where all the information about the recognizable events is stored, and the system behaves as a "parser" matching the incoming data with predefined templates found in the knowledge base. Because of the nature of the explicit event recognition task, it is not surprising that most works on this topic are based on stochastic parsers for the identification of known patterns of atomic events, as in the works by Ivanov and Bobick [8], Minnen et al. [9] or Moore and Essa [10]. Similar to a parser-based approach is the work of $\mathrm{Vu}$, Brémond and Thonnat [11], even if in their case the language used to express complex events is not a full grammar, but rather a set of sub- 
events together with temporal and logical constraints on the subevents. Other works are based on more general stochastic models, such as Bayesian networks, as in Hongeng, Nevatia and Brémond [12] or Moënne-Loccoz, Brémond and Thonnat [13]. More recently, in [14] an unsupervised technique for detecting unusual events in a large video set was presented. In our work we focus on event recognition for the purpose of anomaly detection to support the security personnel.

When persons enter the actual access region their movements are continuously recorded by multiple rotating laserrange scanners. Input data for the person classification are provided by chemical sensors detecting hazardous materials, such as explosives. However, due to the fact that these sensors have a limited spatio-temporal resolution, an individual chemical sensor is unable to localize hazardous material and to associate it with the persons in the surveillance area. Our system realizes an integrative approach that compensates this deficiency in dynamic scenarios by fusing the output of multiple chemical sensors with the kinematic estimates resulting from multiple person tracking based on the laser data (fig. 1 - left).

The incoming laser-range measurements can be assigned to the constructed and successively updated tracks in many ways. Thus, the solution of the assignment problem is crucial for every multiple target tracking algorithm. The traditional approaches to multiple hypothesis tracking rely on the complete enumeration of all possible association interpretations of a series of measurements and avoid an exponential growth of the arising hypothesis trees by various approximations (MHT: Multiple Hypothesis Tracking [22], [23], (J)PDAF: (Joint) Probabilistic Data Association Filter [20]). A powerful, alternative approach is represented by Probabilistic Multiple Hypothesis Tracking (PMHT) [25], [27], [28], [31]. Essentially PMHT is based on Expectation-Maximization for handling assignment conflicts. Linearity in the number of targets and measurements is the main motivation for a further development and extension of this methodology. The original formulation of PMHT [25] deals with measurements that are instantaneous observations of the state of a particular model - here, the kinematical model of a person. The problem of associating measurements to targets arises because the particular model that caused a measurement is unknown. Thus PMHT forms an estimate of the unknown model states based on the state observation with uncertain origin. In practical applications, a sensor may be able to get other information besides the state observations. Davey [24] considers the case where the tracking filter has an estimate of the class of the target that caused each available state observation and extended the PMHT for dealing with classification measurements (PMHT-c). A classification measurement is treated as an observation of the assignment of the corresponding measurement. One example for such a measurement is the range profile that occurs in high resolution radar. However, in our security scenario there is no fixed assignment between a position measurement and a chemical output. In this work we will show how PMHT-c can nevertheless be applied for the purpose of person classification.

The dashed parts in figure 1 (right) are not yet realized within our real-time demonstrator. They refer to the fusion of the event recognition with the chemical person classification. At the current stage the computer vision module and the person classification are running separately.

The remainder of this work is organized as follows: In section II the core components of the proposed security assistance system are introduced. First the computer vision module will be explained. Hereupon we present the state of the art in the development of chemical sensor devices detecting the explosive TATP. In the last part of this section we describe the algorithm that fuses chemical attributes with person tracks and provides the person classification. The classification ability of the algorithm is shown within a simulated scenario. Section III deals with the realization of the demonstrator that was designed to show the operability of the presented concept and to explore the behavior of chemical sensors. We have to point out that the sensors of section II-B are not able to identify TATP in an open system yet. Our current demonstrator is equipped with metal oxide (MOx) sensors detecting hydrocarbons like fuels, alcohols or solvents. Section IV provides a conclusion and a preview of our future work.

\section{CORE COMPONENTS}

\section{A. Video Event Analysis}

The developed computer-vision module has been focused on anomalous event detection for human operator support in security-oriented applications. The acquired video sequence is processed in order to infer a plausible semantic interpretation of the scene, in which the scene itself and its sub-components are labeled with semantic information giving a high-level description of the recognized scenario.

Our solution provides a first level of computation concerning the extraction of moving objects (blobs) from video streams and the computation of relevant features for event analysis purposes. Moving objects are detected from the background (background modeling and foreground segmentation) and their movements tracked on a 2D top-view map of the monitored scene [15]. Tracking is performed using a Kalman Filter applied on map positions together with a Meanshiftbased tracking technique on the image plane [16]. The object classification is performed by employing an adaptive high order neural tree (AHNT) classifier [17] that enables a distinction by two main classes: a) person, b) luggage. At each time instant, a set of low-level features (i.e. object class, position, trajectory, mean speed, etc.) is extracted and maintained for any foreground object in the scene. Once all the necessary features have been extracted from the video stream, an approach based on explicit modeling of dangerous events is adopted to describe and understand the activities occurring inside the monitored environment. Two different types of events have been considered: simple events, characterized by the motion (and behavior) of a single object and composite events, characterized by interactions among multiple objects.

In an indoor environment, a simple event is normally represented by a person or a light vehicle moving in the monitored environment. A simple event $v$ is defined over 
a temporal interval $\left[T^{s}, T^{f}\right]$ and contains a set of features $F=\left\{f_{1}, \ldots, f_{m}\right\}$ belonging to a given object $O_{j}$ observed over a sequence of $n$ consecutive frames as:

$$
v\left(T^{s}, T^{f}\right)=\left\{f_{k} \mid f_{k} \in O_{j}, k \in[1 . . m]\right\}
$$

Composite events are represented by a set of simple events that are spatially and/or temporally correlated. Hence, a composite event is defined over a wide temporal interval as a graph $G(V, E)$ where the set of vertexes $V=$ $\left\{v_{1}\left(T^{s}, T^{f}\right), \ldots, v_{n}\left(T^{s}, T^{f}\right)\right\}$ is the set of simple events and the set of edges $E$ is the set of the temporal and spatial associations between simple events. In the proposed solution we restrict the event association to a set of compatible simple events. This is achieved by exploiting an Event Correlation Diagram (ECD) that describes the allowed relations between object types, their states and actions. It therefore defines the possible links between different simple events, even when they are generated by different objects. To generate the ECD, the explicitly defined simple events are considered. For each of these, its possible relations with any other defined simple event are analyzed and, if any exists, a link between the two simple events is added in the ECD [18].

To recognize composite events thus by associating them to a predefined list of event of interest, we use a graphmatching technique [19]. Each event stored in a graph forest (the database of event of interests) is associated to an alarm level describing the degree of importance of that event in a specific contest. We identified four different alarm levels, in increasing scale of danger. The alarm levels are defined as: a) normal, b) suspicious and c) critical. The 'normal' level is associated to all those events representing a complete event detected within a scene that do not pose any threat from a security point of view. The 'suspicious' level is associated to all those events that are not necessarily dangerous, but that could lead a human operator to identify the threat if the person classification component of our system raised an alarm concerning the same object. In the specific case, a person waiting in front of the control corridor for more than a predefined time is an example of a suspicious event, since generally people have no reason to wait before entering the demonstrator. Hence, if this person is inside the corridor when a chemical alarm raises we can assume that he or she is one of the most probable people to look for. Finally, the 'critical' level is associated to all those events that have been explicitly classified as security threats by the developers of the event knowledge base, and that require an immediate intervention by human operators.

Thus, fusing the alarm level raised by the video event analyzer with those raised by the person classifier inside the tunnel would yield a more robust information about the current threat level for a human operator.

\section{B. Chemical Sensors}

The detection of explosives or explosive related compounds is a challenging task, because most explosives have a very low vapor pressure and do not evaporate enough analyte molecules into air. An exception is TATP (Triacetone Triperoxide), which is known as a homemade explosive often used by terrorists and which has a relatively high vapor pressure. A novel chemical sensor device based on quartz micro balances (QMB) has been developed to trace TATP. Since no exclusive affinity material was found, an array of minimum 3 QMB sensors has to be employed providing data which are unequivocally interpreted by PCA.

The development of efficient sensor materials on a trace level relies on selective binding of the corresponding substrate by well defined molecular recognition sites or distinct affinities [1]. QMB technology can be used as highly sensitive balance which translates very small mass changes typically in the order of nanograms. According to the Sauerbrey equation the frequency shift of a quartz crystal resonator is directly proportional to the added mass [2], [3]. Since the sensitivity is strongly dependent on the fundamental frequency of such resonators, $200 \mathrm{MHz}$ systems were applied in this project. Interactions between thin organic layers deposited on the surface of such a quartz crystal and analytes in headspace result in a mass increment that lowers the fundamental resonance frequency of the oscillating crystal. An inverse modification of its resonance frequency is easily recorded by standard tools [4]. The vapor pressure of TATP (fig. 3) is at ambient conditions in the range of $68 \mathrm{ppm}$ (Vol) [5]. Consequently,

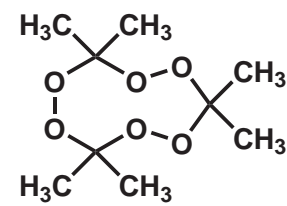

Figure 3. Triacetone Triperoxide (TATP)

enough analyte should be available in the atmosphere to trace TATP. A typical bomb accounts to multi-kilogram quantities of TATP mostly carried in open plastic bags. Since the developed QMB device represents a low cost sensor a mass application is possible. Furthermore, the signal of the QMB is provided almost immediately after exposure to analyte and the sensor device recovers quickly upon removal of target. These are all splendid criteria for an employment within our person classification algorithm presented in the next section. Extensive screening studies of naturally occurring as well as synthetic compounds have led to three selective affinity materials that are appropriate for the detection of TATP with respect to common interfering components. The employed affinity materials belong to salts of bile acid, cyclodextrins, and phenylene dendrimers. The latter have a high affinity for TATP and other polar organic analytes, whereas the bile acid interacts with compounds by hydrogen bridges (esp. hydrogen peroxide or water). Cyclodextrin derivatives exhibit a strong interaction with polar organic compounds such as acetone. Immobilization of the affine coating on QMB discs was accomplished by means of the electrostatic spray method [6]. For tapping the full potential of QMB and getting a 


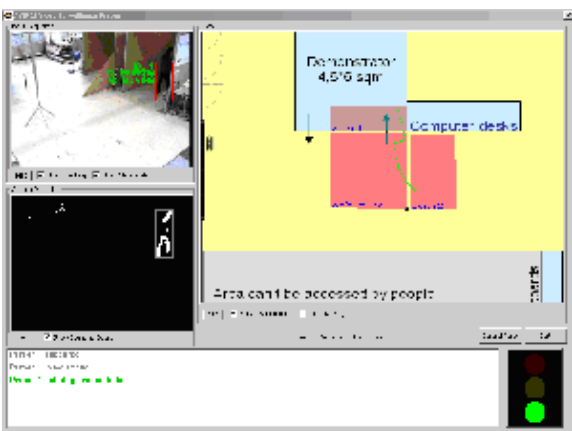

(a)

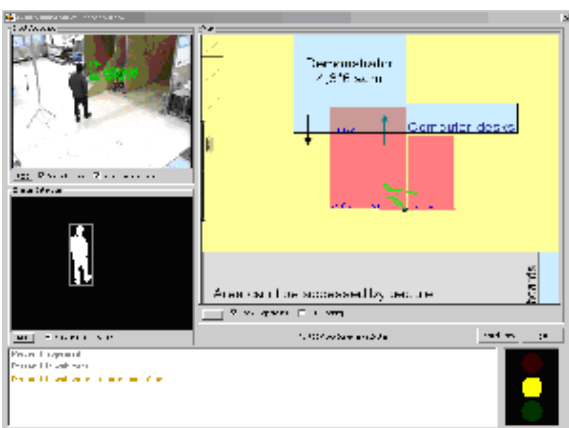

(b)

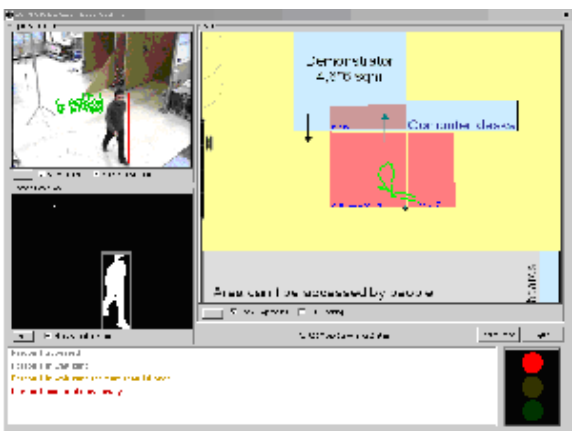

(c)

Figure 2. Example of video event understanding. (a) typical normal event recognized by the system and presented to the human operator with a green light. A suspicious event (b) has been recognized and signaled to the operator with a yellow light. The operator can look in the $\log$ of the events at the bottom of the interface to identify the suspicious entity and investigate more its behavior with respect to the other sensors. Finally a critical event (c) has been recognized and signalled to the operator with a red light. The operator can extract from the event logs the identity of the entity and task other people to deeply investigate.

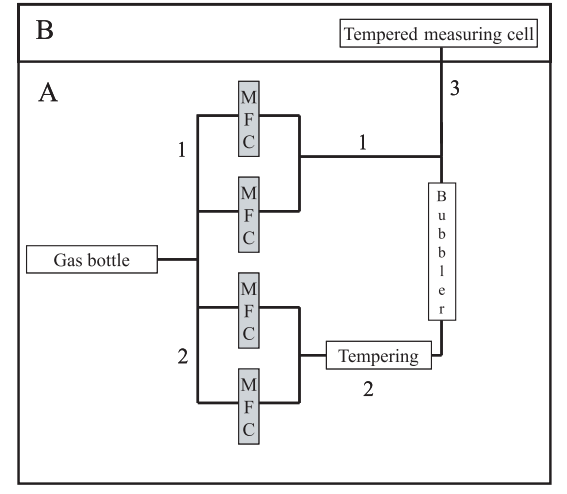

Figure 4. Schematic drawing of calibration setup

practical solution the knowledge of the detection limit is necessary. The test setup has to ensure defined test conditions with respect to temperature and analyte concentration. The

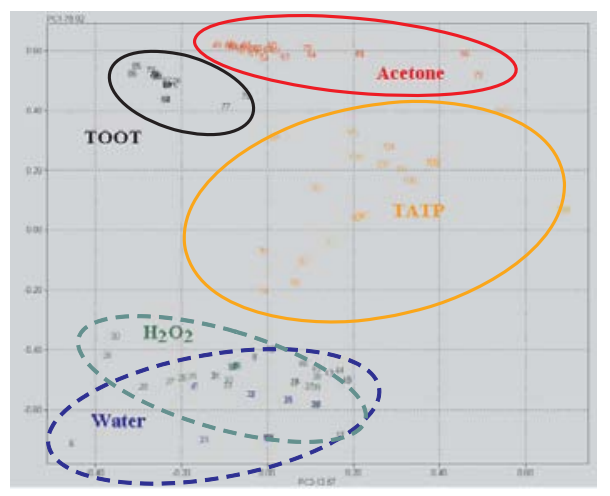

Figure 5. Principal Component Analysis

experimental setup consists of two parts (fig. 4). Part A is a gas mixing unit that creates a well defined gas mixture. Part B consists of an oven, with a multi-sensor array of six QMB inside. Both parts are connected with a short pipe. The feasible TATP concentration range by this method is $3-44$ ppm (Vol) Principal Component Analysis (PCA) of the data set yields two dimensional presentation (fig. 5). The TATP area (orange) is clearly separated from all other competing analytes. An unequivocal detection and identification of TATP was possible even at concentrations as low as 3 ppm (Vol) [7]. However, in the open system TATP cannot be clearly identified yet.

\section{Combined Person Tracking and Classification}

We assume that $S$ persons are moving in the surveillance area and are observed by multiple laser-range scanners. The sensors generate a measurement series $\mathcal{Z}=\mathcal{Z}_{1: T}$ for a time interval $[1: T]^{*}$. The sensor output at a scan $t$ consists of the measurement set $\mathbf{z}_{t}$ (containing the measurements of all sensors) and of the number of all measurements $N_{t}$. Measurements $\mathbf{z}_{t}^{n} \in \mathbb{R}^{2}$ with $n \in\left[1: N_{t}\right]$ are assumed to be Cartesian position data. The task of person-tracking consists in estimating the kinematic states $\mathcal{X}=\mathcal{X}_{1: T}$ of the observed persons (the person tracks). The states $\mathbf{x}_{t}^{s} \in \mathbb{R}^{4}$ with $s \in[1: S]$ comprise position and velocity. Each person moves according to a discrete-linear model [21]. Difficulties arise from unknown associations $\mathcal{A}=\mathcal{A}_{1: T}$ of measurements to persons. The associations are modeled as random variables $a_{t}^{n}$ that map each measurement $n \in\left[1: N_{t}\right]$ to one of the persons $s \in[1: S]$ by assigning $a_{t}^{n}=s$.

1) Multiple Person Tracking: Probabilistic multiple hypothesis tracking (PMHT) is an efficient method to solve the tracking problem. It works on a sliding data window (also called batch) and exploits the information of previous and following scans in every of its kinematic state estimates. For each window position, the method of expectation-maximization (EM) [26] is applied to the underlying data. Based on EM, an iterative algorithm can be derived [25]. Let $l$ be the number of the current iteration. Each iteration consists of two steps.

Starting with the Expectation-Step (E-Step) we calculate posterior assignment weights $p\left(a_{t}^{n}=s \mid \mathbf{z}_{t}^{n}, \mathbf{x}_{t}^{s}(l)\right)$ representing

\footnotetext{
* $[1: T]$ denotes the integral interval from 1 up to $T$
} 
the probability that a measurement $\mathbf{z}_{t}^{n}$ refers to a person $s$. The weights are calculated for all scans of the current data window and for all persons with respect to all measurements of a certain scan. Each weight is governed by the distance between a particular measurement $\mathbf{z}_{t}^{n}$ and the state estimate $\mathbf{x}_{t}^{s}(l)$. Hereupon the weights are used to form the weighted sum $\overline{\mathbf{z}}_{t}^{s}(l)$ of all measurements which leads to one synthetic measurement per person at each scan $t$. There is another formula for the corresponding synthetic covariance. During the Maximization-Step (M-Step) each person track is updated by means of a Kalman Smoother that processes the synthetic values. This leads to new, improved state estimates $\mathbf{x}_{1: T}^{s}(l+1)$ for each person $s$.

E-Step and M-Step are repeated until the state estimates do not change considerably anymore (convergence). After convergence, the prediction $\mathbf{x}_{T+1 \mid T}^{S}$ is to be calculated for the following window position. When all persons have been processed, the window is shifted by one scan and the iteration process is started for the new window position. Detailed formulae of the PMHT algorithm are presented in [29].

2) Incorporating Classification Information: Originally PMHT-c [24] was designed to take advantage of classification measurements to improve data association and state estimation. In the considered scenarios, the class observation could be utilized to improve tracking, because for each position measurement the corresponding classification output was known. The author deals with pairs of measurements that consist of a kinematical and a classification part. The target class estimates occur as a by-product. High resolution radar is one example of a system where these classification measurements exist. Range profiles from various azimuth angles form a radar image of the target. The location of primary scatterers can be used to classify the target.

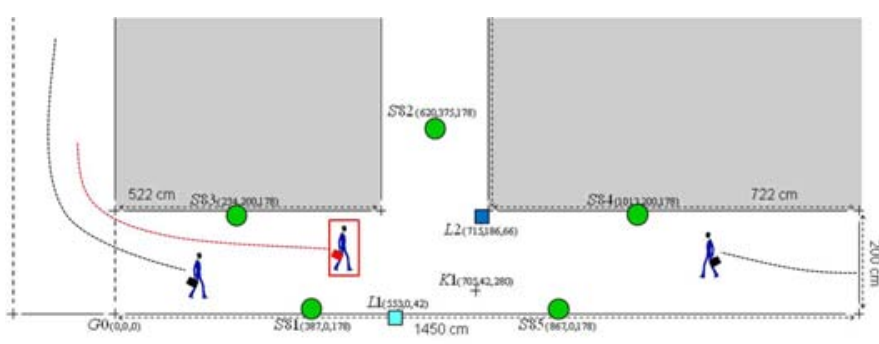

Figure 6. Corridor with 5 Chemos (circles) and 2 Lasers (rectangles)

In our system there is no information about the assignment of chemical attributes to laser data. To apply PMHT-c we have to consider the scenario in a different way: In the security scenario there are also pairs of position and classification output but this position is not provided by the lasers. In fact it is given by the chemical sensor placement and output. So referring to the experimental corridor in figure 6 we have five measurement pairs at each scan $t$. Each of them consists of the chemical sensor position and its classification output. In the following we explain how to associate the chemical outputs with person tracks applying PMHT-c and to find out who is carrying the hazardous material.

In an early version of our algorithm [30] we used the installation place of each chemical sensor as its position information. The closer a person passes a chemical sensor, the higher is the influence of the reported sensor output with respect the classification of this person. The procedure fails if another person, not carrying an explosive, stays closer to the sensor than the dangerous one. A precise mathematical model of a chemical sensor could yield more useful position information, e.g. an estimated distance of the chemical source based on the amplitude of the chemical signal. In this case the position measurement belonging to a certain classification output lies on a circle whose radius is determined by the value of the signal amplitude (fig. 7).

Let $\mathbf{p}^{\text {ch }}$ denote the position information of the chemical sensor $c h \in[1: 5]$ ( $s$ and $t$ omitted for the sake of simplicity). We denote the classification measurement associated with $\mathrm{p}^{\text {ch }}$ as $o_{t}^{\text {ch }}$ and let the total measurement vector $\mathbf{z}_{t}^{\mathrm{ch}}:=\left(\mathbf{p}^{\mathrm{ch}}, o_{t}^{\mathrm{ch}}\right)^{\top}$ be the collection of the position (state observation) with its associated classification measurement. The classification measurement is a discrete variable that can take a value from five different classes: green stands for No Alert and yellow, orange, red and dark red symbolize the alert levels from I up to IV ( $\equiv$ low to high). Our problem can be formalized as follows: Given the estimated kinematic states $\mathcal{X}^{l}$ of the $S$ persons we want to estimate their classification. The desired information is represented by a so called confusion matrix $\mathrm{C}=\left\{c_{i s}\right\}$ whereas the entry $c_{i s}$ is the probability that person $s$ produces class output $i$.

Starting from these modeling assumptions and observations the PMHT-c algorithm [24] can be applied. To get PMHT$\mathrm{c}$, the expectation-step (E-Step) and the maximization-step (M-Step) of the basic PMHT have to be extended by the classification estimates.

a) Calculate Assignment Weights (E-Step): First we have to calculate the posterior assignment probabilities $w_{t}^{\mathrm{ch} \rightarrow s}(l)$. Following [24] we use the update formula

$$
w_{t}^{\mathrm{ch} \rightarrow s}(l)=\sigma \cdot \mathcal{N}\left(\mathbf{p}^{\mathrm{ch}} ; \mathbf{H x}_{t}^{s}(l), \mathrm{Cov}\right) \cdot c_{o_{t}^{\text {ch }} s}(l) .
$$

These posterior weights reflect the relevance of a chemical output for a person $s$ in the surveillance area. The posterior assignment weights are mainly governed by the Gaussian $\mathcal{N}\left(\mathbf{p}^{\text {ch }} ; \mathbf{H} \mathbf{x}_{t}^{s}(l)\right.$, Cov $)$, which is a measure for the distance between the position information of sensor ch and the current position estimate of person $s$ (fig. $7-$ top). $\mathbf{H}$ is the observation matrix. The corresponding covariance matrix Cov reflects the uncertainty of the position information and has to be experimentally determined. $c_{o_{t}^{\text {ch }}}(l)$ is the current estimate of the matrix entry that associates the output of sensor ch with person $s$. The posterior weights $w_{t}^{\mathrm{ch} \rightarrow s}(l)$ are calculated for each sensor ch and each person s at each scan of the current data window.

b) Update Classification Matrix (M-Step): During the M-Step our parameter estimates have to be updated. Besides the parameters for tracking purposes, we have to update the 
entries of the classification matrix. Following [24] this means processing formula (3).

$$
c_{i s}(l+1)=\frac{\sum_{t=1}^{T} \sum_{\mathrm{ch}=1}^{5}\left(\delta\left(o_{t}^{\mathrm{ch}}-i\right) \cdot w_{t}^{\mathrm{ch} \rightarrow s}(l)\right)}{\sum_{t=1}^{T} \sum_{\mathrm{ch}=1}^{5} w_{t}^{\mathrm{ch} \rightarrow s}(l)}
$$

As PMHT-c works on a sliding data window, not only the relevance weights of the current scan are available, but also the whole history inside the time window can be taken into account and evaluated (fig. 7 - bottom). To update the

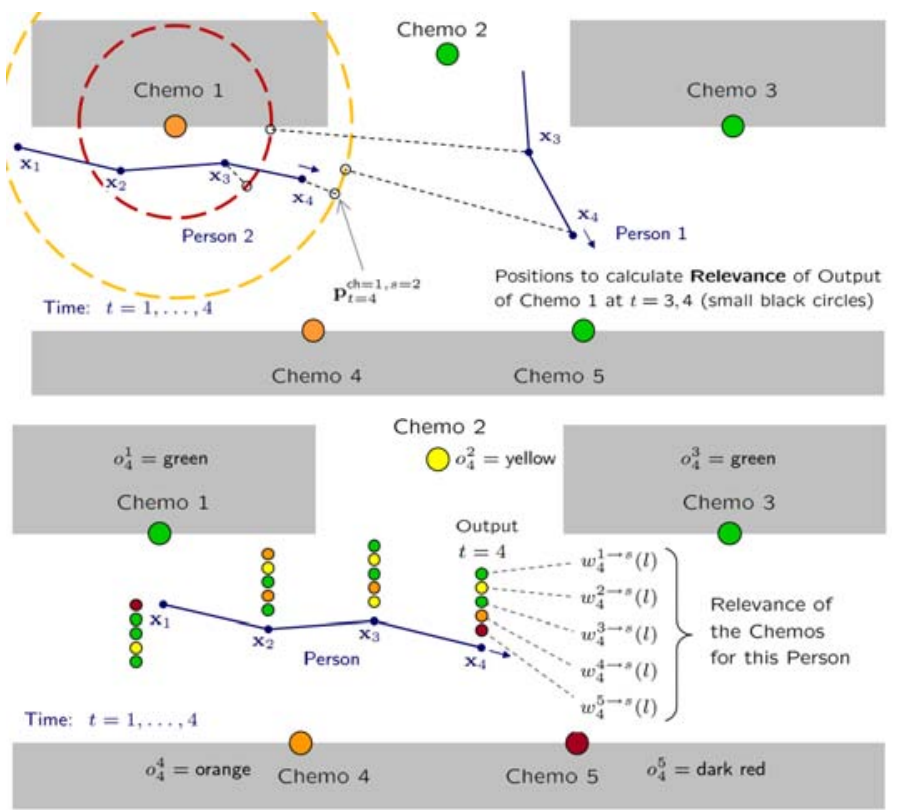

Figure 7. E-Step (top) and M-Step (bottom) of the classification

classification entry for a certain alert level $\in$ \{green, yellow, orange, red, dark red $\}$ one has to sum up all posterior weights of sensors that indicate this level. The weights have to be normalized with respect to the whole window (eq. 3).

c) Simulations: The classification ability of PMHT-c is shown in a simulation (fig. 8): Two persons are walking from the left to the right entry of the corridor. We calculate the sum of all alert probabilities, that is $\sum_{i=\mathrm{I}}^{\mathrm{IV}} c_{i s}$ for a person $s$, and renormalize these sums with respect to the persons (number at the track heads). The dangerous person is marked by a dark gray rectangle. The chemical sensors react according to the Euclidian distance of the person and since we are in a simulated world, the behavior of the chemical sensors is well understood and absolutely reliable. The sensor model provides a suitable source distance for every chemical output. The solid line of the plots corresponds to the numbers plotted at the head of the tracks. The black dashed line is the value before renormalization and the four colored dashed-dotted lines show the values of the alert entries in the confusion matrix.
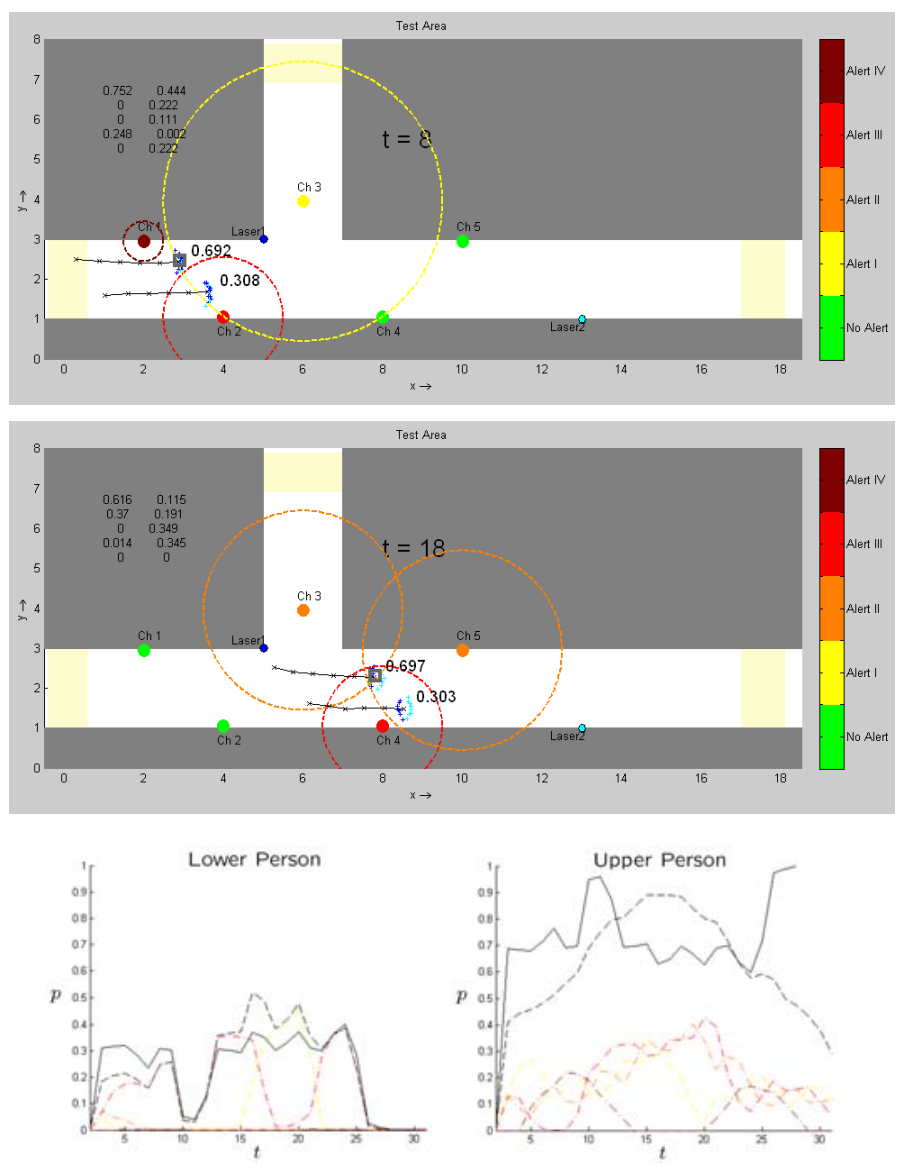

Figure 8. Simulation - Upper person carries material

\section{Design of THE Demonstrator}

The real-time demonstrator was used for both characterization of the chemical sensor response and demonstration of the operability of the overall concept. We designed it as a corridor containing a U-turn (fig. 9). The aisle has a width of $1.89 \mathrm{~m}$, a height of $2.50 \mathrm{~m}$ and a total length of $9 \mathrm{~m}$. Ceiling and walls are made of low-emission press board mounted on an adjustable metal frame. The tracking data are derived from three laser-range scanners which are connected to PC1. Two types of chemical sensors are installed: Firstly, there are five MOx-sensors which are connected with PC2. They deliver the chemical attributes for the data fusion, i.e. for the test of the PMHT based classification algorithm (sec. II-C). Secondly, we installed two QMB-sensors, which are run separately on PC3. This setup was used to test the applicability of the currently available QMB-technology (sec. II-B).

All sensors are integrated into the air inlet sides of an adjustable air ventilation system (fig. 9). The sucking side consists of one air ventilator $\left(V_{\max }=1060 \mathrm{~m}^{3} / \mathrm{h}\right)$, which is connected to four inlet tubes of $100 \mathrm{~mm}$ diameter, which are $1.36 \mathrm{~m}$ above ground. The airflow of the inlets can be adjusted as a whole and separately. On the opposite wall from the air inlets, corresponding air blowers $\left(V_{\max }=175 \mathrm{~m}^{3} / \mathrm{h}\right.$ each $)$ are installed. The throughput of each air blower can be adjusted 
separately and different sizes of nozzles can be attached. The direction of the forced airflow is indicated by the blue arrows (fig. 9 - top). Position, size and design of both air blower and air inlet can be completely altered by adjusting the press board walls accordingly. The video system is mounted on a $4 \mathrm{~m}$-long pole approximately $6 \mathrm{~m}$ outside of the demonstrator entrance and connected to PC4. For our demonstration purposes, we
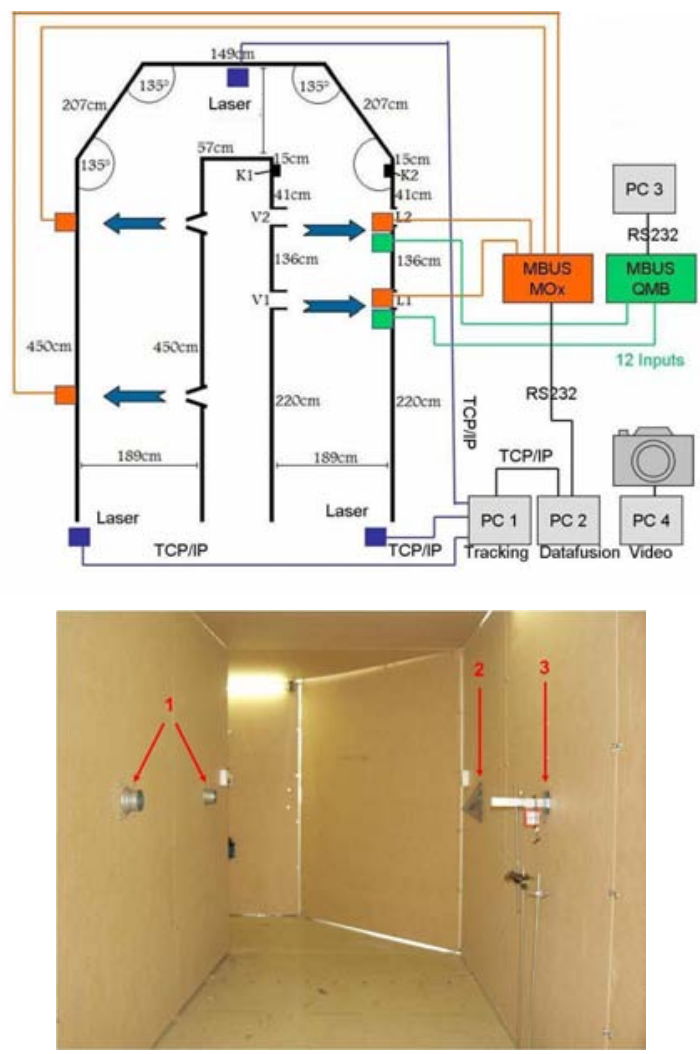

Figure 9. Design and interior view of the real-time system

used chemical sensors for hydrocarbons like fuels, alcohols or solvents. The installed metal oxide (MOx) sensors are sensitive devices for the detection of these analytes. The surface resistance of a thin film of metal oxide particles (mostly tin dioxide) decreases if a small amount of the analyte (usually a few parts per million or less) is present in ambient air. The higher the concentration of the analyte the higher is the sensor signal.

After processing a multitude of tests, we were able to empirically analyze the MOx sensors. Figure 10 shows the averaged sensor signal when a person passes it at five different distances. To get the complete sensor model, we made an area interpolation with cubic splines. The plot clearly shows the high delay of about $10 \mathrm{sec}$ at each of the distances $(\approx 6 \mathrm{sec}$ with ventilation). However, both a reliable determination of the delay and the derivation of the source distance from a given signal amplitude emerged as a troublesome problem. The values in figure 10 are averaged and they can vary from time to time in an unpredictable way which makes the correct classification difficult. Nevertheless we were able to achieve some good results when the persons were walking well separated (fig. 11).

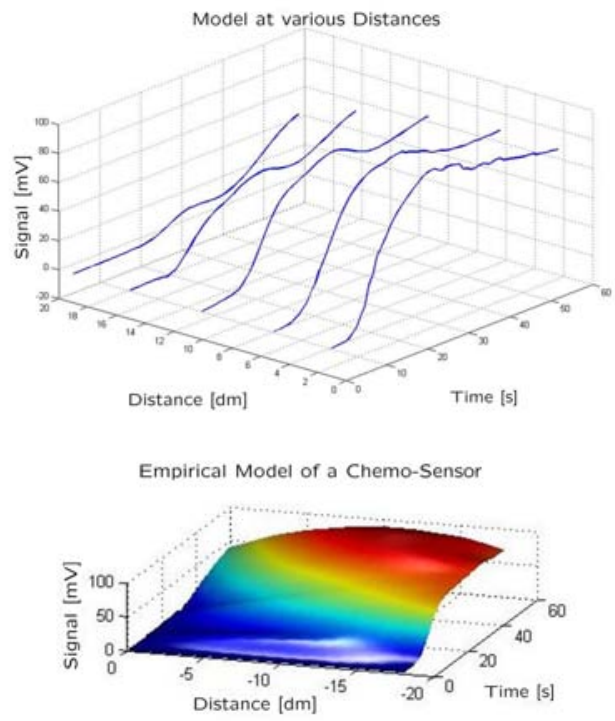

Figure 10. Empirical model of a MOx sensor
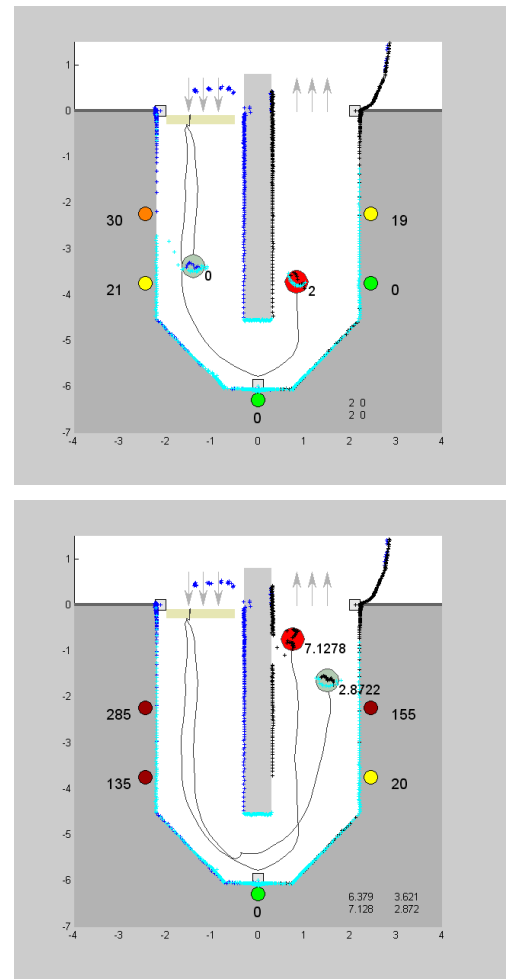

Figure 11. Snapshots of the real-time system

\section{SUMmARY AND CONCLUSIONS}

We presented the concept and the realization of a security assistance system based on video surveillance and fusion of person tracks with chemical sensor data. A computer vision module provides a video event analysis of the entrance region 
of the system. Inside the system, the entering persons are scanned by multiple rotating laser devices and the estimated person tracks are fused with the output of multiple chemical sensors detecting hazardous materials.

Sensor systems based on quartz micro balances (QMB) with chemically modified surfaces are a powerful method to detect the explosive TATP and other hazardous and volatile analytes. With a sensor array, TATP can be unequivocally identified in a few seconds. However, in the open system TATP cannot be clearly identified yet. Systems which allow the tracing of TATP in an open system are current work.

The classification ability of the PMHT-c algorithm could be successfully shown under simulated conditions, i.e. constant delay and functional dependency of source distance and sensor output. Nevertheless, the tests within our real-time environment showed that many more experiments will be necessary to understand the reaction of a chemical sensor and to find a suitable mathematical model. Analyte diffusion, convection and transportation by the ventilation air stream influence the gas concentration and should be taken into account.

Furthermore the fusion of the chemical person classification with the video event analysis seems to be a promising approach. It is already included in the concept presented in this paper (fig. 1) and will be a topic of our future research.

\section{REFERENCES}

[1] J. Janata (Ed.), Special Issue on Modern Topics in Chemical Sensing, Chem. Rev., vol. 108, pp. 327-844, 2008.

[2] B. Neubig, and W. Brise, Das große Quarzkochbuch, FranzisVerlag, Feldkirchen, 1997.

[3] G. Sauerbrey, The use of quartz oscillators for weighing thin layers and for microweighing, Z. Phys. vol. 155, pp. 206-222, 1959.

[4] J. Bargon, B. Graewe, T. Jonischkeit, and K. Woelk, Sensorik Von der Waage zur elektronischen Nase, Chem. Unserer Zeit, vol. 37, pp. 212-213, 2003.

[5] J. C. Oxley, J. L. Smith, K. Shinde, J. Moran, Determination of the vapor density of triacetone triperoxide (TATP) using a gas chromatography headspace technique, Propellants, Explosives, Pyrotechnics, vol. 30, pp. 127-130, 2005.

[6] C. Heil, G. R. Windscheif, S. Braschohs, J. Floerke, J. Glaeser, M. Lopez, J. Mueller-Albrecht, U. Schramm, J. Bargon, and F. Voegtle, Highly selective sensor materials for discriminating carbonyl compounds in the gas phase using quartz microbalances, Sens. Actuators B, vol. 61, pp. 51-58, 1999.

[7] S. R. Waldvogel, J. W. Lörgen, D. Lubczyk, K. Müllen, and R. Bauer, Novel Device for the on-line Detection of TATP, DE1020080086606, 2008.

[8] Y. Ivanov and A. Bobick, "Recognition of visual activities and interactions by stochastic parsing," IEEE Trans. on Pattern Analysis and Machine Intelligence, vol. 22, no. 8, pp. 852-872, 2000.

[9] D. Minnen, I. Essa, and T. Starner, "Expectation grammars: leveraging high-level expectations for activity recognition," in Computer Vision and Pattern Recognition, 2003, pp. II-626-632.

[10] D. Moore and I. Essa, "Recognizing multitasked activities using stochastic context-free grammar," in IEEE Int. Conf. con Computer Vision and Pattern Recognition, Kauai, HI, USA, 2001.

[11] V. Vu, F. Brémond, and M. Thonnat, "Automatic video interpretation: A novel algorithm for temporal scenario recognition," in 8th International Joint Conference on Artificial Intelligence, Acapulco, MEX, 2003, pp. 9-15.
[12] S. Hongeng, R. Nevatia, and F. Brémond, "Video based event recognition: activity representation and probabilistic methods," Computer Vision and Image Understanding, vol. 96, pp. 129162, 2004.

[13] N. Moenne-Loccoz, F. Brémond, and M. Thonnat, "Recurrent bayesian network for the recognition of human behaviors from video," in Int. Conf. On Computer Vision Systems, Graz, Austria, 2003, pp. 68-78.

[14] H. Zhong, J. Shi, and M. Visontai, "Detecting unusual activity in video," in Proceedings of the IEEE Computer Society Conference on Computer Vision and Pattern Recognition, vol. 2, Washington DC, U.S.A, 27 June - 2 July 2004, pp. 819-826.

[15] G. L. Foresti, C. Micheloni, L. Snidaro, P. Remagnino, and T. Ellis, "Active video-based surveillance systems," IEEE Signal Processing Magazine, vol. 22, no. 2, pp. 25-37, March 2005.

[16] D. Comaniciu, V. Ramesh, and P. Meer, "Kernel-based object tracking," IEEE Transactions on Pattern Analysis Machine Intelligence, vol. 25, no. 5, pp. 564-575, May 2003.

[17] G. Foresti and T. Dolso, "Adaptive high-order neural trees for pattern recognition," IEEE Transactions on System, Man and Cybernetics Part B, vol. 34, no. 2, pp. 988-996, Apr. 2004.

[18] C. Micheloni, L. Snidaro, and G. L. Foresti, "Statistical event analysis for video surveillance," in Proceedings of the Sixth IEEE Internationl Workshop on Video Surveillance, Graz, Austria, May $13^{\text {th }}$ 2006, pp. 81-88.

[19] D. Conte, P. Foggia, C. Sansone, and M. Vento, "Thirty years of graph matching in pattern recognition," International Journal of Pattern Recognition and Artificial Intelligence, vol. 18, no. 3, pp. 265-298, 2004.

[20] Y. Bar-Shalom and T. E. Fortmann, Tracking and Data Association, Academic Press, 1988.

[21] Y. Bar-Shalom, X. R. Li and T. Kirubarajan, Estimation with Applications to Tracking and Navigation, John Wiley \& Sons, Inc., 2001.

[22] S. S. Blackman and R. Populi, Design and Analysis of Modern Tracking Systems, Artech House, Boston (USA), 1999.

[23] S. S. Blackman, Multiple-Target Tracking with Radar Applications, Artech House, Boston (USA), 1986.

[24] S. J. Davey, Extensions to the Probabilistic Multi-Hypothesis Tracker for Improved Data Association, University of Adelaide, Dissertation, 2003.

[25] R. L. Streit and T. E. Luginbuhl, "Probabilistic MultiHypothesis Tracking," Naval Undersea Warefare Center Division, NUWC-NPT/10/428, 1995.

[26] M. A. Tanner, Tools for Statistical Inference. Location: Springer Verlag, Berlin (Germany), 1996.

[27] M. Wieneke and W. Koch, "The PMHT: Solutions for Some of its Problems," SPIE - Conferences of Optics and Photonics (Signal and Data Processing of Small Targets), San Diego (USA), 2007.

[28] M. Wieneke, W. Koch, "On Sequential Track Extraction using Expectation-Maximization," EURASIP, special issue "Track Before Detect Algorithms", 2008.

[29] M. Wieneke, K. Safenreiter and W. Koch, "Combined Person Tracking and Classification in a Network of Chemical Sensors," ISIF $-11^{\text {th }}$ International Conference on Information Fusion, 2008.

[30] M. Wieneke, K. Safenreiter and W. Koch, "Hazardous Material Localization and Person Tracking," SPIE - Conferences of Optics and Photonics (Signal and Data Processing of Small Targets), Orlando (USA), 2008.

[31] P. K. Willett, Y. Ruan and R. L. Streit, "PMHT: Problems and Some Solutions," IEEE Transactions on Aerospace and Electronic Systems, vol. 38 , no. 3 , pp. 738-754, 2002. 